\title{
Long term clinical benefits of pancreas-transplantation after kidney transplantation in patients with diabetes who had received kidney transplantation
}

\author{
Youngmin Ko
}

Department of Surgery-Transplantation, Asan Medical Center, University of Ulsan College of Medicine, Seoul, Korea

Background: The purpose of this study is to prove long term clinical benefits of pancreas-transplantation after kidney transplantation (PAK) in patients with diabetes who had received kidney transplantation.

Methods: Data from patients who had kidney transplantation from January 2009 through July 2020 at Asan Medical Center were retrospectively analyzed. Of 1,042 consecutive patients, 21 patients underwent PAK. Kidney graft failure, biopsy-proven acute rejection of kidney, and patient survival were compared between the two groups (PAK vs. non-PAK).

Results: There was no significant difference in baseline characteristics between the two groups. During 10-year follow-up, biopsy proven acute rejection rate in the PAK group was significantly higher compared with the control group $(P=0.006)$. There was no difference in the incidences of kidney graft failure and patient survival between two groups.

Conclusions: It seems that there is a higher risk of biopsy-proven acute rejection of kidney graft in recipients who received PAK.

Corresponding author: Youngmin Ko

E-mail: chun8710@naver.com

\footnotetext{
(c) The Korean Society for Transplantation

This is an Open Access article distributed under the terms of the Creative Commons Attribution Non-Commercial License (http://creativecommons.org/licenses/by-nc/4.0/) which permits unrestricted non-commercial use, distribution, and reproduction in any medium, provided the original work is properly cited.
} 